Original Research

\title{
The Relationship Between Vegetarian Diet and The Risk of Coronary Heart Disease
}

\author{
R. I. Gunadi ${ }^{1}$, K. Shonafi ${ }^{1}$, R. Bagus ${ }^{1}$, A. Andrianto ${ }^{1,2^{*}}$ \\ ${ }^{1}$ Faculty of Medicine, Universitas Airlangga, Surabaya, Indonesia. \\ ${ }^{2}$ Department of Cardiology and Vascular Medicine, Soetomo General Hospital, Surabaya, Indonesia.
}

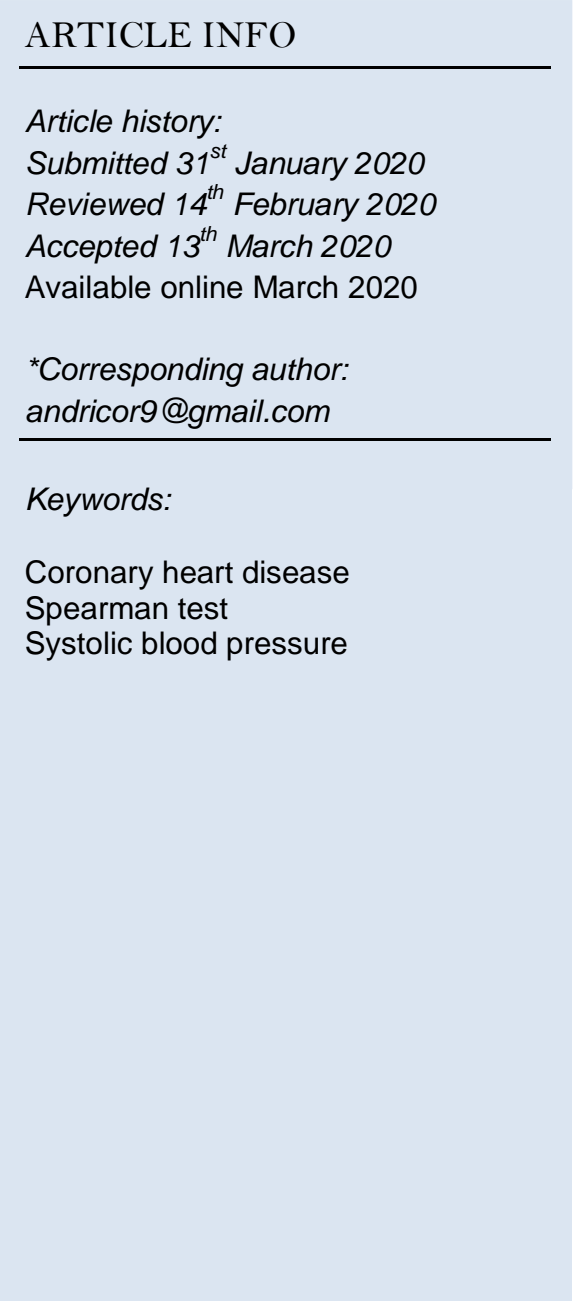

\section{ABSTRACT}

Background. Coronary heart disease (CHD) is a major cause of death in developed countries whereas most cases can be prevented by addressing behavioral risk factors, such as unhealthy diet. Vegetarian dietary patterns reduce CHD risk and mortality by $40 \%$. The Framingham Heart Study on Hard CHD calculates a 10-year risk assessment with six predictors, two of which are lipid profile types. Aims. To discover the relationship between vegetarian diet and the risk of coronary heart disease, we did a cross-sectional analytical observation in North Jakarta using 30 healthy vegetarians and 30 healthy non-vegetarians aged of 20-34 years in 2010 . Framingham Heart Study on Hard CHD 10-year risk assessments were done on both groups. Methods. Results were compared and analyzed using Mann-Whitney $U$ and Spearman correlation tests. Results. Mann-Whitney $U$ test showed significant differences on total cholesterol and total FHS risk points ( $p$-value 0.000 ; pvalue 0.000 ). Spearman correlation analysis on total risk points confirms a moderate negative degree for vegetarian diet and $\mathrm{CHD}$ risk $(r=-$ 0.525, $p$-value 0.000). In conclusion, there is a significant relationship between vegetarian diet and a reduced risk of coronary heart disease in 20-34 years of age.

\section{Introduction}

Cardiovascular disease (CVD) contributes a large number of growing public health epidemic in noncommunicable disease (NCD) and burden in people under the age of 70 years in low and middleincome countries (LMICs). There is substantial concord that NCDs, including CVD, are majorly associated with four behavioral risk factors: poor diet, physical inactivity, tobacco use, and excessive alcohol use ${ }^{[1]}$. Healthy behavioral habits may reduce the risk of myocardial infarction by more than $80 \%$, with nutrition playing a pivotal role. Developing a vegetarian diet may minimize the risk of coronary heart disease (CHD) and CVD mortality by $40 \%{ }^{[2]}$

Besides their no meat diet, most vegetarians live with other healthy life style habits such as abstinence from smoking and alcohol consumption, 
and regular workout physical activities. Studies on risk factors of chronic disease conclude that compared to non-vegetarians, vegetarians have lower serum cholesterol concentrations, lower body mass indices, lower incidence of diabetes and possibly lower blood pressure.

Huang et al conducted a meta-analysis comprised of 124,706 participants from 7 studies, which included lacto-ovo vegetarians (A no-meat or fish diet, but do eat eggs or dairy products, or both) or vegans (no meat, fish, eggs or dairy products). In a previous study, six studies were included in the analysis of ischemic heart disease mortality and found that ischemic heart disease mortality was significantly lower (by 29\%) in vegetarians than in non-vegetarians $(\mathrm{RR}=0.71 ; 95 \% \mathrm{Cl}, 0.56-0.87)$. Previous study concluded that the reduced total cholesterol serum, oxidation of low density lipoprotein cholesterol (LDL-C), relatively low prevalence of smoking and alcohol consumption in these legion contributed to the low ischemic heart disease mortality rate among the vegetarians. ${ }^{[3]}$

The Framingham Heart Study (FHS) on hard coronary heart disease perfectly summarizes and calculates the risk factors that contribute to CHD, especially myocardial infarction or coronary death. It consists of six predictors: age, total serum cholesterol, high-density lipoprotein cholesterol (HDL-C), systolic blood pressure (SBP), treatment for hypertension and smoking status, with a duration of follow up maximum of 12 years and risk calculated at ten years ${ }^{[4,5]}$. End results of this calculation are the sum of all predictors scores, known as the total FHS risk assessment points. Lower total points are associated with a lower 10year risk for hard CHD. This study aims to analyze the relationship of $\mathrm{CHD}$ and vegetarian patterns compared to non-vegetarians in a 20-34 year population in North Jakarta using the Framingham
Heart Study on Hard CHD as our central risk assessment calculating tool.

\section{Methods}

A cross-sectional observational analysis was done in 30 healthy vegetarians and 30 health nonvegetarians in the age range of 20-34 years. Sample was taken by purposive sampling, with all vegetarian samples taken from a Vihara/Buddhist Temple in North Jakarta, and non-vegetarian samples were medical students studying at Atma Jaya Medical Faculty in North Jakarta. The inclusion criteria for vegetarians were: have minimally undergone a vegetarian diet for at least one year, a citizen of Jakarta, aged 20-34 years and gave their informed consent. Exclusion criteria were: any prior history of cardiovascular disease, diabetes, kidney disease, hypertension, hyperlipidemia, and also undertaking antihypertensive or cholesterol medication drugs, including oral/hormonal contraceptives. Inclusion and exclusion criteria were adjusted and applied to non-vegetarians as well.

\section{Results}

The respondents were 15 male and 15 female in both groups, in the non-vegetarian group mean age is $20.57(20-22)$ years as of the vegetarians is 26.73 (20-34) years. All respondents were nonsmoking and did not have a history of smoking. After applying normality tests on five variables (age, systolic blood pressure, total cholesterol, HDL-C, and total Framingham Heart Study (FHS) risk assessment) results confirmed that age, SBP, and total FHS risk assessment points did not have a normal distribution. Hence, a non-parametric statistical analysis (Mann-Whitney $U$ ) was used further (as seen on Table 1). 
Table 1. Comparison of variable significance between non-vegetarian and vegetarian groups.

\begin{tabular}{lccc}
\hline & Non-Vegetarian & Vegetarian & p-value \\
\hline Age & $2.57 \pm 0.57$ & $26.73 \pm 4.07$ & $0.000^{\mathrm{a}}$ \\
SBP & $117.83 \pm 9.78$ & $118.00 \pm 9.88$ & 0.904 \\
Total Cholesterol & $199.17 \pm 30.88$ & $164.77 \pm 17.20$ & $0.000^{\mathrm{b}}$ \\
HDL Cholestrerol & $52.67 \pm 3.56$ & $52.87 \pm 3.82$ & 0.835 \\
Total FHS Risk & $-1.80 \pm 2.84$ & $-4.80 \pm 2.02$ & $0.000^{\mathrm{a}}$ \\
Assessment Points & & \\
\hline Note & & \\
SBP : Systolic Blood Pressure & FHS : Framingham Heart Study & \\
${ }^{\mathrm{a} p}$ value by independent samples test & b $\mathrm{p}$ value by Mann-Whitney U test & \\
\hline
\end{tabular}

Mann-Whitney $U$ calculations were significant $(p-$ value 0.000 ) for age and total FHS risk assessment points, whereas systolic blood pressure proved no significance. As for total cholesterol and HDL-C, both had a normal distribution and so underwent a parametric-statistical analysis, using independent sample test. Total cholesterol proved to be significant ( $p$-value 0.000), but HDL-C had no significant difference. To better understand the correlation of vegetarian and non-vegetarian diets and total FHS risk assessment points, Spearman correlation analysis was done. All statistic calculations were done using SPSS Statistics Ver.24.

Table 2. Correlation results using Spearman analysis

\begin{tabular}{lcc}
\hline \multicolumn{1}{c}{ Variables } & R & p-value \\
\hline Vegetarian Status - Total FHS Risk Assessment Points & -0.525 & 0.000 \\
\hline FHS : Framingham Heart Study & & \\
\hline
\end{tabular}

From table 2 above, we can identify that vegetarian status have a significant and moderate to strong inverse correlation with Total FHS Risk Assessment Points.

\section{Discussion}

Based on the division of National Cholesterol Education Program (NCEP) Expert Panel on Detection, Evaluation, and Treatment of High Blood Cholesterol in Adults (Adult Treatment Panel III), the risk of $\mathrm{CHD}$ divided into 3 categories, namely 0 1 risk factor, $2+$ risk factor $(\leq 20 \%)$, and $\mathrm{CHD}$ or $\mathrm{CHD}$ risk equivalents (10-year risk more than $20 \%)^{[6]}$. After the Framingham Heart Study risk assessment calculated data on all variables, all respondents in both groups are in the 0-1 risk factor category. Even though results were much equal or uniform on both groups, we found a significant difference of vegetarian dietary patterns on three variables, namely age, total cholesterol, and total FHS risk assessment points. Using Spearman correlation analysis, we also found that vegetarian diet had a significant negative correlation direction with total FHS risk assessment points ( $p$-value $0.000)$ with moderate strength $(r=-0.525)$. One of our study's inclusion criteria was that a vegetarian respondent must have undergone a vegetarian diet for at least one year, and in that minimal 
requirement, the effect has already made a significant impact. These results have stated that even in young aged population, maintaining a healthy diet and lifestyle is profoundly substantial in reducing cardiovascular events, more so if one continues to pursue a vegetarian diet.

The results of our study are consistent with other studies on vegetarian dietary pattern and $\mathrm{CHD}$ incidence and mortality. Most studies agree that a vegetarian diet can reduce CHD incidence and mortality through a series of beneficial control in substantial risk factors such as normal body weight (BMI), blood lipids, and blood pressure ${ }^{[7,8]}$. Eleven trials in a meta-analysis and systematic review done by Wang et al. stated that vegetarian diets significantly lowered blood concentrations of total cholesterol with a pooled estimation $-0.36 \mathrm{mmol} / \mathrm{L}$ $(95 \% \mathrm{Cl}-0.55 \text { to }-0.17 ; \mathrm{P}<0.001)^{[9]}$. A systematic review done by Gary E. Fraser states that lower total cholesterol and LDL-C is the main outcome of a consistent vegetarian diet, most possibly achieved as animal saturated fats raise LDL-C levels and most vegetarians avoid meat and animal products ${ }^{[8]}$. Fraser also concludes that a vegetarian dietary pattern does not give a significant impact on HDL-C level, which our study concurs. We did not find any significant differences in HDL-C level between vegetarian and non-vegetarian groups.

Although the study results may provide some useful information, several limitations should be considered. Our study results have limited sampling methods and amount; we performed a purposive sampling method, which may apprehend the results with confounding and subjectivity factors. Our sample was taken only in one institution on each group (non-vegetarians in medical students at Atma Jaya University, North Jakarta; and vegetarians in members of the Maitreya Wira Vihara/Buddhist Temple, North Jakarta). Our study also lacks a sufficient amount of each member groups (nonvegetarian and vegetarian) to represent the population of North Jakarta

\section{Conclusion}

In conclusion, there is a significant correlation between vegetarian diet and a reduced total Framingham Heart Study risk assessment points in 20-34 years of age, this may be due to significantly lower total blood cholesterol levels in vegetarian group. These total points will be useful in predicting 10-year risk of hard coronary heart disease, where lower total points have a lower 10-year risk of CHD.

\section{Acknowledgement}

There is no conflict of interest.

\section{References}

1. Yeates K, Lohfield L, Sleeth J, Morales F, Rajkotia $\mathrm{Y}$ and Ogedegbe O. 2015. A global perspective on cardiovscular disease in vulnerable populations. HHS Public Access. Can. J. Cardiol; 31, 9: 1081-93.

2. Kahleova H, Levin S and Barnard N D 2018 Vegetarian Dietary Patterns and Cardiovascular Disease Prog. Cardiovasc. Dis. 61(1):54-61

3. Huang $T$ and Wahlqvist $L 2012$ Cardiovascular Disease Mortality and Cancer Incidence in Vegetarians: A Meta-Analysis and Systematic Review Ann. Nutr. Metab. 60:233-40

4. Sanchis-gomar F, Perez-quilis $C$, Leischik $R$ and Lucia A 2016 Epidemiology of coronary heart disease and acute coronary syndrome Ann. Transl. Med. 4:256-68.

5. US Department of Health and Human Services 2013 Assessing Cardiovascular Risk: systematic evidence review from the risk assessment work group.

6. Expert Panel on Detection, Evaluation and Treatment of High Blood Cholesterol in Adults. 2001. Executive Summary of the Third Report 
(NCEP) Expert Panel on Detection, Evaluation, and Treatment of High Blood Cholesterol in Adults (Adult Treatment Panel III). JAMA; 285: 2489-97.

7. Dauchet L, Amouyel P and Dallongeville J 2009 Fruits, vegetables and coronary heart disease Nat. Publ. Gr. 6:599-608.
8. Fraser G E 2009 Vegetarian diets : what do we know of their effects on common chronic diseases?. Am. J Clin.Nutr. 89:1607-13.

9. Wang F, Zheng J, Yang B, Jiang J, Fu Y, and Li D. 2015. Effects of vegetarian diets on blood lipids: A systematic review and meta-analysis of randomized controlled trials. J Am Heart Association; 4: 1-14. 